\title{
The relation between post concussion symptoms and neurocognitive performance in concussed athletes
}

\author{
Vanessa C. Fazio, Mark R. Lovell*, Jamie E. Pardini and Michael W. Collins \\ University of Pittsburgh Medical Center, Department of Orthopaedics, Pittsburgh, PA, USA
}

\begin{abstract}
The objective of this study was to examine differences in neurocognitive performance between symptomatic concussed athletes, a group of concussed athletes with no subjective symptoms, and a non-concussed control group of athletes. All concussed athletes were evaluated within one week of injury using the ImPACT computerized test battery. Results indicate that concussed athletes who denied subjective symptoms demonstrated poorer performance than control subjects on all four composite scores of the ImPACT test batters (Verbal Memory, Visual Memory, Reaction Time and Processing Speed However, the concussed but asymptomatic group demonstrated significantly better performance than did the concussed and symptomatic group. Thus, concussed athletes who did not report subjective symptoms were not fully recovered based on neurocognitive testing. This study underscores the importance of neurocognitive testing in the assessment of concussion sequelae and recovery.
\end{abstract}

Keywords: Concussion, mTBI, symptoms

\section{Introduction}

The International Symposium on Concussion in Sports has acknowledged the role of neuropsychological testing in concussion assessment and return to play considerations [3,32]. The initial guidelines set forth at the $1^{\text {st }}$ international symposium in Vienna, Austria detailed a "comprehensive systematic approach to concussion injury" which included clinical history, evaluation, neuropsychological testing, imaging procedures, research methods, management and rehabilitation, prevention, education, future directions, and medical/legal considerations [3]. The guidelines advocated the acute use of brief neuropsychological batteries to assess attention and memory function with more extensive and specific testing conducted post injury. The Vienna Concussion in Sports group (CIS) declared neuropsycho-

*Address for correspondence: Mark R. Lovell, Ph.D., ABPN, UPMC Sports Medicine Concussion Program, 3200 S. Water Street, Pittsburgh, PA 15203, USA. Tel.: +1 412432 3681; E-mail: lovellmr @upmc.edu. logical testing to be "the cornerstone of concussion evaluation" and stated that it "contributes significantly to both the understanding of the injury and the management of the individual" [3, p. 8].

A subsequent statement from the CIS about the value of neuropsychological testing in concussion management was not as forceful, but, nevertheless, still proclaimed it to be an "aid to clinical decision making" as part of a comprehensive management system [32]. The follow-up conference in Prague, Czech Republic in 2004 proposed a new classification system for concussion and a new, brief, measurement tool for the acute assessment of concussion. However, the importance of neuropsychological testing to determine cognitive recovery in concussion was again underscored [32].

Evidence supporting the use of neurocognitive testing has come from numerous studies that have examined its clinical utility. The initial impetus for the utilization of neuropsychological testing in sports came from the publication of a large-scale multi-center study by Barth and his colleagues in 1989 [6]. This study used pre-season (baseline) testing as a means of evaluating post-injury changes in a group of collegiate ath- 
letes . Another major step in the clinical utilization of neurocognitive testing involved the adoption of an evaluation program by the National Football League in $1994[21,35,38]$ and the National Hockey League in 1997 [22]. More recently, neurocognitive testing has become increasingly commonplace in collegiate $[10$, 12,23] and high school athletes [24,34].

Further evidence of the importance of neuropsychological testing in concussion assessment and management has been found in studies of athletes with multiple concussion histories and learning disorders. Athletes with a history of multiple concussions ( 2 or more) or learning disabilities do not perform as well as normative groups in baseline testing. This underscored the importance of having an accurate assessment of premorbid neurocognitive functioning to accurately measure impairment related to concussion [10]. Ravdin, Barr, Jordan, Lathan, and Relkin hypothesized several other factors that may affect baseline assessment scores in boxers involved in intense training [41]. Similar to the Collins et al. study with football athletes, Ravdin and colleagues found that a history of multiple concussions in boxers was linked to decreased neuropsychological test performance post injury, specifically in the area of verbal memory [41].

\subsection{Concussion symptoms}

Common signs and symptoms of concussion have been well documented and researched in the sports concussion literature. Diagnosis of sport-related concussion is made when an athlete demonstrates any mental status change following a traumatic force to the head or body. Mental status changes can include loss of consciousness (usually brief), retrograde amnesia, anterograde amnesia, and confusion/disorientation. In addition to mental status changes, concussion typically produces a constellation of symptoms that is usually shortlived in nature and resolves on its own over time. Of the common concussion symptoms, headache is most often reported, and is closely followed by fatigue, feeling slowed down, drowsiness, and cognitive problems. Other symptoms may include sleep disturbance, personality change, nausea, etc. $[17,28]$. Post-concussion symptom presentation, like general concussion recovery and initial injury presentation, is a very individualized process that can manifest in a variety of ways. Concussed athletes may report one symptom of injury, or many different types of symptoms. Also, symptoms may present immediately after injury in many players, though have delayed onset in others. For example, Mc-
Crea et al. described a small subsample of concussed athletes ( $N=11$ out of 94 ) whose post-injury symptoms appeared, on average, 14.4 minutes after injury, with a standard deviation of 15.5 minutes [30].

Post-concussion symptom presentation has been demonstrated in many studies to vary dependent upon the severity of injury. For example, a study comparing concussed athletes with good versus poor outcomes at 72-hours post-injury revealed that athletes with significantly exacerbated symptoms and memory deficit relative to baseline were approximately ten times more likely to have experienced at least three of four abnormal on-field markers of severity (LOC, disorientation, PTA, RGA). Poor outcome at 72 hours (elevated symptoms and memory problems) was also associated with increased probability of experiencing retrograde amnesia, posttraumatic amnesia, and disorientation as a result of concussion [9]. A similar study compared outcomes from concussed athletes exhibiting one or more on-field mental status changes lasting greater than 5 minutes to outcomes when mental status changes are shorter than 5 minutes in duration. In this study, athletes exhibiting a shorter duration of mental status changes had returned to baseline on both memory performance and symptom status by day 4 post-injury, whereas those with protracted mental status changes had not recovered by the day 7 evaluation. Regardless of on-field mental status changes, all concussed athletes in the sample reported significant increases in symptom reporting relative to baseline at 36 hours, though not at days 4 and 7 [25]. In a study of college football players McCrea et al. found that concussed athletes continued to report significantly greater amount of symptoms compared to their own baseline symptoms up to day 7 post-injury [30] Patterns of post-injury cognitive impairment were similar, with most deficits resolving by day 7 as well . However, even at day 90 post-injury, concussed players performed more poorly than matched controls on a verbal fluency measure.

Recent research has also explored the importance of individual symptoms in concussion recovery. Headache is the most frequently reported postconcussion symptom, and has been the most widely researched as well. Studies have reported a wide range for the incidence of headache following concussion, ranging from $40 \%$ to $86 \%[15,28,31]$. Presence of postconcussion headache, regardless of severity, has been associated with higher levels of post-concussion symptomatology, memory dysfunction, and slowed reaction times at 1 week after injury [9]. Processing speed was not differentially affected by headache status in that 
study. When the headache group was subdivided into mild and moderate/severe groupings, those with more severe headaches reported a significantly higher number of other post-concussion symptoms than those in the mild group. With regard to on-field markers of injury, athletes reporting headache at the post-injury evaluation were significantly more likely to have experienced on-field anterograde amnesia, and were almost 5 times more likely to have demonstrated any onfield mental status change lasting greater than 5 minutes [9]. Another study examining the role of headache in concussion divided concussed athletes into three groups: non-headache (N-HA), headache (HA), and headache with post-traumatic migraine characteristics (PTM) [33]. Concussed athletes with PTM symptoms demonstrated the greatest post-injury impairment, with significantly lower scores on visual memory, verbal memory, processing speed, and reaction time scores at approximately day 4 post-injury when compared to the HA and N-HA groups. Similarly, the HA group demonstrated significantly lower scores when compared to the N-HA group at the post-injury assessment. Similar patterns were observed with symptom reporting.

Many clinicians who evaluate and manage sportsrelated concussions have heard athletes report feeling mentally "foggy" or "hazy" as a result of the injury. In fact, this sensation was frequently reported such that one author (MRL) incorporated the symptom of "feeling foggy" into the Post-Concussion Symptom Scale (PCS) [23]. Sideline assessment tools, such as the newly released Sport Concussion Assessment Tool (SCAT) also incorporate items assessing a "foggy" or "dazed" feeling [32]. Recently, Iverson and colleagues examined the relationship between athletes' report of fogginess and outcome at 5 to 10 days post-injury [18]. Similar to findings in the headache studies, athletes endorsing any degree of fogginess reported a significantly higher number of other concussion-related symptoms, and demonstrated significantly worse performance on memory, reaction time, and processing speed measures.

\subsection{The relationship of subjective symptom reporting to neurocognitive testing}

Symptom report and resolution is an important factor in concussion assessment; however, neuropsychological testing may continue to reveal subtle lingering deficits even after symptom resolution. Furthermore, self-reported symptoms and neurocognitive test performance may not always agree [32]. For instance, Peterson, Ferrara, Mrazik, Piland, and Elliott found symptoms and vestibular ratio (as measured through the Smart Balance Master System) were similar to controls at the third day post concussion while neuropsychological test results continued to remain significantly different than controls at 10 days post injury [39]. Duration of symptoms has been found to be significantly related to decreased neuropsychological test scores as well [13]. The evidence of the interplay of symptoms and neuropsychological testing underscored statements of the CIS regarding the use of multiple modalities to assess and manage concussion injury [3,32].

Despite a general acknowledgment of the utility of neurocognitive testing in helping to make return to play decisions, the precise "value added" of neuropsychological assessment in addition to symptoms report has not been demonstrated until recently. Van Kampen et al. found that symptom report alone only identified $65 \%$ of concussed athletes two days post injury as compared to $83 \%$ identified by neuropsychological testing alone [44]. Together, symptom report and neuropsychological testing identified $93 \%$ percent of concussed athletes. Therefore, the use of both symptoms and neurocognitive testing could increase the identification of concussed athletes by over twenty five percent.

\subsection{Computerized assessment of cognitive and symptom deficits}

The endorsement of neuropsychological testing for professional, collegiate, and adolescent/child athletes has enhanced the management of concussion, but also created a quandary for those administering and interpreting test results $[3,32,35]$. Despite the utility of testing, the administration of "paper and pencil" neuropsychological tests is time consuming, costly, requires administration by a psychometrist or neuropsychologist, interpretation by a neuropsychologist, and is nearly impossible to administer in large group settings [42]. Additionally, the lack of sensitivity and specificity to mild neuropsychological changes that occur in concussion and normative data that only differentiates the "grossly impaired" make many traditional "paper and pencil" neuropsychological tests inadequate for concussion assessment [8,42]. Recent development of computerized neuropsychological testing designed to assess sports concussion has alleviated some of these concerns, and has increased the availability and feasibility of baseline and post injury neurocognitive testing for athletes.

The ImPACT (Immediate Post-concussion Assessment and Cognitive Testing) battery is one computerized test battery that can be used in concussion assess- 
ment. ImPACT encompasses both symptom assessment via the Post-Concussion Symptom Scale (PCS) and evaluation of four domains of neurocognitive functioning: verbal memory, visual memory, visual motor processing speed, and reaction time [27,29]. Table 1 provides a description of the tests which comprise the composite scores.

These four domains are assessed through six neuropsychological tests that target attention, memory, processing speed, and reaction time $[18,24,25,40]$. The Post-Concussion Symptom scale is included to assess 21 concussion-related symptoms as rated on a 6 point severity scale (PCS) [23].

Research evaluating the validity and reliability of change using ImPACT has shown this battery to be an effective tool in concussion assessment and management $[19,29,35,42]$. Processing speed and reaction time composites on ImPACT were found to correlate highly with a standard neuropsychological "paper and pencil" test of these domains [19]. Moreover, test retest coefficients for ImPACT composite scores indicated a high degree of reliability $(0.65-0.86)$ that is similar to other neuropsychological tests [20].

Most recently, research regarding the sensitivity and specificity of the ImPACT instrument has indicated its utility as part of a formal concussion management program. Schatz, Pardini, Lovell, Collins, and Podell found the combination of PCSS, ImPACT Processing Speed composite, and ImPACT Visual Memory composite correctly identified $82 \%$ of concussed athletes [42]. Furthermore, ImPACT was found to have an $89.4 \%$ positive predictive value (PPV - probability that a concussion is present if the test is positive) and an $81.9 \%$ negative predictive value (NPV - probability that a concussion is not present when the test is negative). Overall, results of the study suggested that the sensitivity and specificity (discriminating power) of ImPACT is satisfactory [42].

\subsection{Objective}

The purpose of this study was to explore the relation between post concussion neurocognitive functioning and symptom presentation. While a relationship between symptoms reported and neurocognitive sequelae has been indicated in the literature, Peterson et al. demonstrated that neurocognitive impairment may still be present even after symptoms have remitted [39]. Therefore, it is likely that impaired neuropsychological functioning may persist in concussed athletes who have been formally diagnosed as having sustained a concussion but do not present with symptoms one to three days post-concussion.

The present study examined between-group differences in neuropsychological performance of concussed symptomatic, concussed-asymptomatic, and control participants. We hypothesized that there would be significant differences in neurocognitive test results between the three groups post-injury. More specifically, it was hypothesized that concussed-symptomatic athletes would demonstrate the most impaired performance and concussed-asymptomatic athletes would display cognitive impairment relative to controls.

\section{Methods}

\subsection{Participants}

This study was approved by the University of Pittsburgh Institutional Review Board. The study analyzed the data of 192 participants (78 concussed symptomatic athletes, 44 concussed asymptomatic athletes and 70 non-concussed control athletes). Table 2 provides important demographic information regarding these three groups. Criteria for inclusion in the concussion group required that the concussed high school or collegiate athletes were tested within 7 days of sustaining a concussion. The non-injured control group consisted of high school and collegiate athletes who underwent baseline ImPACT testing and were then retested within 2 days of the initial assessment. Concussed athletes participated primarily in football while controls participated primarily in non-contact sports such as track and swimming. Participants in the injury groups were likely to be male, and were not significantly different with regard to age $(F=1.89, p<0.15)$.

As part of the Sports Medicine Concussion Program at the University of Pittsburgh Medical Center, all athletes in this study underwent a baseline or pre-injury evaluation, and were administered ImPACT before the 2001 to 2004 athletic seasons. Following a concussion (as diagnosed by a physician or certified athletic trainer present at the time of injury), all concussed athletes received follow-up testing with the ImPACT battery within seven days of injury, with most athletes being evaluated within two days of injury (mean $=1.88$ days, range $=1$ to 7 days). The non-concussed control group was tested an average of 13 days (range $=0-48$ days) after the initial baseline testing to provide the basis for comparison and to help control for practice effects on testing due to prior exposure to the test battery. 
Table 1

ImPACT Neurocognitive Test Battery

\begin{tabular}{|c|c|}
\hline Test Name & Neurocognitive Domain Measured \\
\hline Word Memory & Verbal recognition memory (learning and retention) \\
\hline Design Memory & Spatial recognition memory (learning and retention) \\
\hline$X$ 's and O's & Visual working memory and cognitive speed \\
\hline Symbol Match & Memory and visual-motor speed \\
\hline Color Match & Impulse inhibition and visual-motor speed \\
\hline Three letter memory & Verbal working memory and cognitive speed \\
\hline Symptom Scale & Rating of individual self-reported symptoms \\
\hline Composite Scores & Contributing Scores \\
\hline Verbal Memory & $\begin{array}{l}\text { Averaged percent correct scores for the Word Memory (learning and delayed), Symbol Match memory test, Three } \\
\text { letters memory test }\end{array}$ \\
\hline Visual Memory & Averaged percent correct scores for the Design Memory (learning and delayed), X's and O's test \\
\hline Reaction Time & $\begin{array}{l}\text { Mean time in milliseconds for the X's and O's test (average counted correct reaction time), Symbol Match (average } \\
\text { weighted reaction time for correct responses), Color Match (average reaction time for correct response) }\end{array}$ \\
\hline $\begin{array}{l}\text { Visual Motor } \\
\text { Processing Speed }\end{array}$ & $\begin{array}{l}\text { X's and O's (average correct distracters), } \\
\text { Symbol Match (average correct responses) } \\
\text { Three Letters (number of correct numbers correctly counted) }\end{array}$ \\
\hline
\end{tabular}

Table 2

Demographic data for the symptomatic, asymptomatic and control samples

\begin{tabular}{lccc}
\hline Variable & Symptomatic & Asymptomatic & Control Subjects \\
\hline $\mathrm{N}=$ & 78 & 44 & 70 \\
Mean (range) age (yrs) & $16.7(12-27)$ & $16.6(14-22)$ & $17.3(14-22)$ \\
Mean (range) education (yrs) & $10.2(8-15)$ & $10.2(8-15)$ & $10.9(8-16)$ \\
$\quad$ High school & $80 \%$ & $85 \%$ & $72 \%$ \\
$\quad$ College & $20 \%$ & $25 \%$ & $28 \%$ \\
Previous concussions & & & \\
0 & $76 \%$ & $77 \%$ & $90 \%$ \\
1 & $14 \%$ & $14 \%$ & $10 \%$ \\
2 & $8 \%$ & $4.5 \%$ & $0 \%$ \\
3 & $2 \%$ & $4.5 \%$ & $0 \%$ \\
Gender: (\% male) & $82 \%$ & $80 \%$ & $47 \%$ \\
Sport & $68 \%$ & $68 \%$ & $0 \%$ \\
American Football & $5 \%$ & $20 \%$ & $24 \%$ \\
Soccer & $13 \%$ & $2 \%$ & $0 \%$ \\
Basketball & $6 \%$ & $5 \%$ & $0 \%$ \\
Wrestling & $0 \%$ & $0 \%$ & $50 \%$ \\
Swimmers & $0 \%$ & $0 \%$ & $17 \%$ \\
Track & $8 \%$ & $5 \%$ & $9 \%$ \\
Other & & & \\
Time between injury to testing & 1.6 days $(0-7$ days) & 2.4 days $(0-7$ days) & 2.5 days $(2-5$ days) \\
(range) & & & \\
\hline
\end{tabular}

\subsection{Materials/procedures}

The ImPACT test battery includes a standardized demographic questionnaire that requires the athlete to document relevant educational, sports participation, and personal medical history. For this study, test results from athletes who had completed ImPACT version 2.0 or later were utilized. Previous versions of ImPACT differed with regard to test composition, and therefore these data were excluded. Test administrators were trained to define concussion as a "traumatically induced alteration in mental status that may or may not be accompanied by a loss of consciousness," based on the standard American Academy of Neurology nomenclature [1].

In-season concussions were diagnosed on the basis of the following criteria [25]: 1) any observable alteration in mental status or consciousness on following a blow to the head or body during sport participation, and/or 2) the presence of LOC and/or anterograde or 
retrograde amnesia identified in an on-field examination, and/or 3) any self-reported symptoms such as cognitive "fogginess," headache, nausea and/or vomiting, dizziness, balance problems, and visual changes after a collision involving the head or body. Certified athletic trainers or team physicians who were present on the sideline at the time of injury made the initial diagnosis of concussion.

\subsection{Analyses}

One-way analyses of variance were conducted to identify between-group differences on age. A MANOVA was conducted to establish between-group differences on the dependent measures (the ImPACT composite scores). All analyses were conducted using SPSS statistical software [43].

\section{Results}

The three groups being studied were not significantly different with regard to age $(F=1.98, p<0.15)$. With regard to prior history of concussion, there was no significant difference between the symptomatic and asymptomatic group $(F=0.001, p<0.97)$ but the control group did have a significantly lower average number of prior concussions compared to the two injured groups $(F=4.4, p<0.01)$.

Multiple Analysis of Variance (MANOVA) was employed to evaluate differences in neuropsychological test performance between the symptomatic, asymptomatic and control groups. The MANOVA provides an overall statistical assessment of whether the three subject groups varied significantly with regard to neurocognitive performance.

Table 3 presents the detailed descriptive statistics for verbal and visual memory, processing speed and reaction time composite scores. Comparisons between symptomatic, asymptomatic and control athletes are illustrated in Figs 1-3.

The overall MANOVA was highly statistically significant $(F=14.5, p<0.001)$. Subsequent univariate analyses were then conducted to identify which specific ImPACT composite scores differed for the three groups. For the verbal memory composite score, there was a significant difference in performance between the three groups, $F=34.4, p<0.00$. Specifically, the concussed-symptomatic group performed most poorly followed by the asymptomatic-concussed group. The control group performed better than the two injured groups.

In terms of visual memory, similar results were found: there was a highly significant difference between the symptomatic, asymptomatic and control groups with the symptomatic group performing most poorly, followed by the asymptomatic and control groups $F=41.6, p<0.00)$. The finding of poorer performance for the symptomatic group, relative to the other two groups was also clearly for the Processing Speed Composite $F=32.4, p<0.00)$ and the Reaction Time Composites $F=33.6, p<0.00$ ).

\section{Discussion}

Sports-related mild traumatic brain injury, which includes concussion, has become a public health concern given the high rate of occurrence and the younger age of the population being affected (5 to 24 years) [11]. Bailes and Cantu recently estimated that the rate of concussion injury in United States football was, at a minimum, 1.5 million injuries per year [4]. Most contact sport participants, and therefore those most at risk for concussion injury, are high school and collegiate athletes. Recent studies have shown that these groups have a slower recovery period and more significant neurocognitive effects from the concussion than professional athletes [5,14,34,36-38].

Given the significant effects concussion injury can have on neurocognitive functioning and academic, social and emotional functioning, proper management of this injury is essential. In addition, recent evidence has suggested that sports-related concussion may lead to changes in vascular regulation, and other neurometabolic processes, that may lead to chronic or lifethreatening consequences such as second impact syndrome $[7,14,16]$.

Current guidelines from the CIS stress the importance of neurocognitive assessment if there is impairment on sideline assessment which includes selfreported symptoms. Therefore, when return to play decisions are made on the field, this decision is likely to be primarily determined by the athlete's symptom report. However, given the well-known tendency of competitive athletes to underreport or minimize symptoms, presumably in hopes of expediting their return to play [36], the over-reliance on self-reported symptoms is likely to result in increased exposure to risk of re-injury. 
Table 3

Group Means and Standard Deviations for ImPACT Composite Scores of Symptomatic, Asymptomatic, and Control

\begin{tabular}{lccc}
\hline ImPACT Composite Score & Symptomatic & Asymptomatic & Control Group \\
\hline Verbal Memory & $73.1(15.7)$ & $79.8(10.6)$ & $89.6(7.7)$ \\
Visual Memory & $60.5(13.8)$ & $67.6(13.5)$ & $80.0(11.7)$ \\
Reaction Time & $0.67(0.13)$ & $0.58(0.08)$ & $0.53(0.06)$ \\
Processing Speed & $32.0(8.9)$ & $37.0(6.5)$ & $42.2(6.9)$ \\
\hline
\end{tabular}

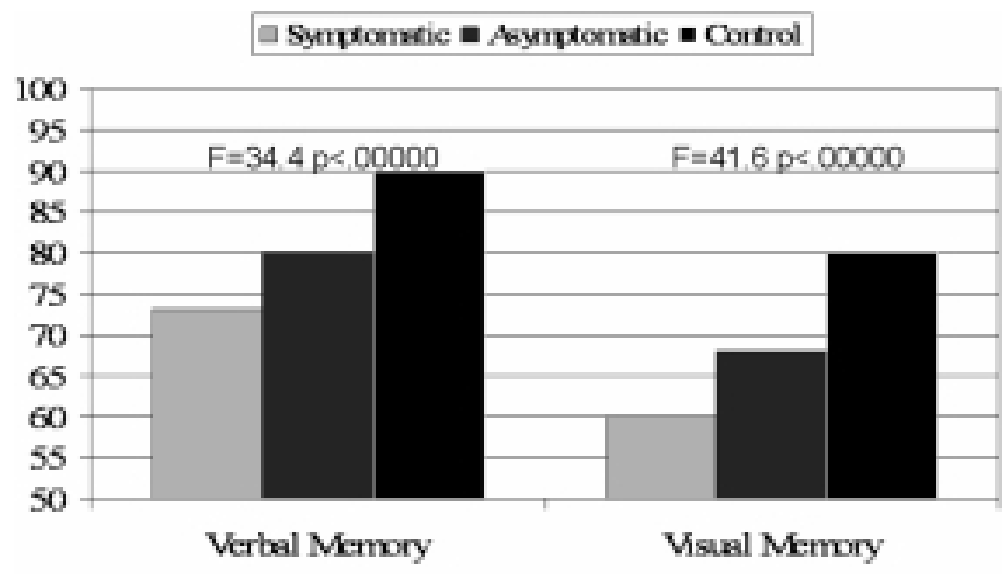

"Neuropsychobogical test performance on ImPACT, a computer based test batteryMemory Composite. Higher ecores indicate better perfommance.

Fig. 1. Unique contribution of neurocognitive testing: Memory composites.

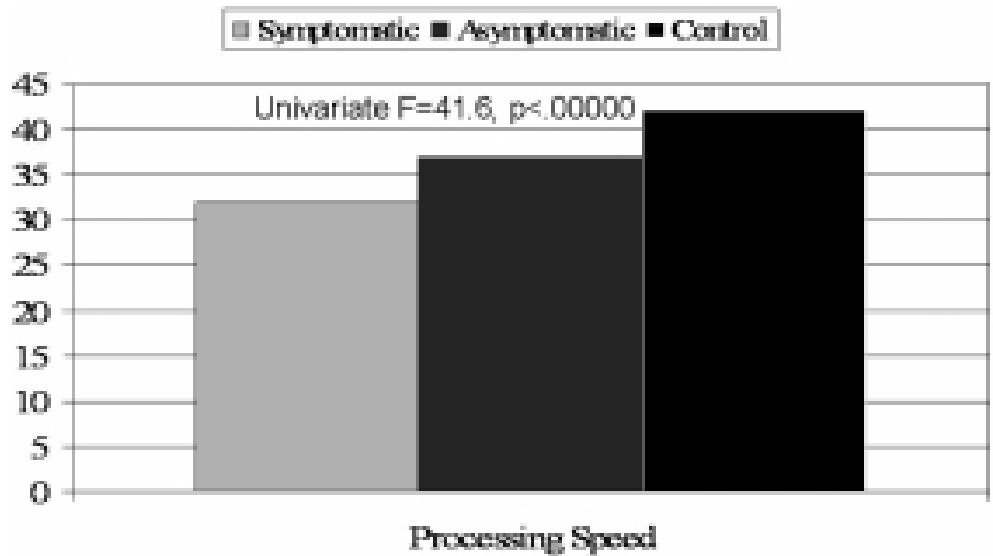

* Nenroprychological test performance on ImPACT, a computer taned test betteryProcesing Speed Composite. Higher noces indseate betler performatioe.

Fig. 2. Unique contribution of neruocognitive testing: Processing speed composite.

The findings of this study are consistent with past studies that have shown an imperfect relationship between symptom reporting and neurocognitive recovery, indicating that neuropsychological assessment may be necessary for even asymptomatic athletes when a concussive event is suspected $[12,36,44]$. While the symp- tomatic group was significantly more impaired than the other groups on all cognitive domains measured by ImPACT, the asymptomatic group still performed worse on these measures than a control group, signifying some lingering impairment to cognitive processes. Additionally, Van Kampen et al. found that symptom report and 


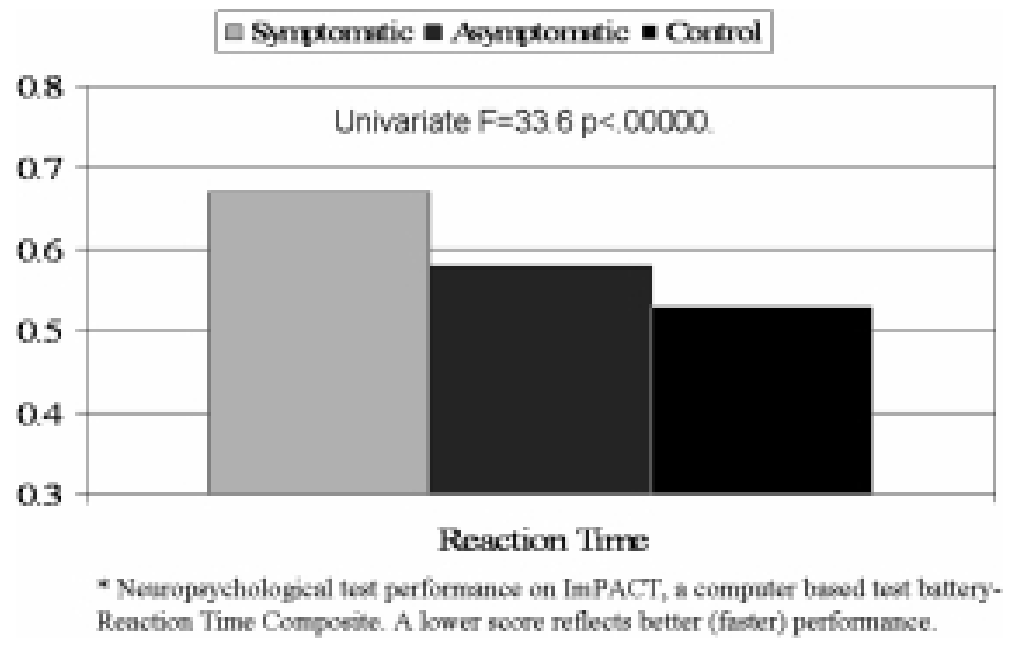

Fig. 3. Unique contribution of neruocognitive testing: Reaction time composite.

neuropsychological testing correctly identified $94 \%$ of concussed athletes whereas symptom report only identified $65 \%$ of these athletes [44]. Therefore, many athletes with concussion may go undiagnosed and unmanaged because they are under-reporting symptoms or are asymptomatic.

Results from this study and corresponding literature on symptom reporting suggest that clinicians should not rely on symptom report alone to determine return to play status. There are many possible reasons athletes may under report symptoms. First, athletes may blatantly deny symptoms due to internal or external pressure to compete. In this regard, the athlete may fear losing his or her position to another team member if he/she is not able to play. In addition, the culture of sports necessitates a certain degree of athletic ability and physical endurance as indicated in many colloquial expressions such as "no pain, no gain" and "you can't make the club in the tub". The value of "endurance" may lead many athletes to view themselves as "weak" if their participation is halted by post concussion symptoms. External pressure from parents, coaches and team members may also lead to a sense that the athlete is "letting the team down" if he or she is not able to compete.

In addition to the blatant denial of symptoms by the athlete, there may be more subtle reasons that symptoms are not accurately reported. For instance, athletes may mislabel or fail to identify symptoms when they occur. This type of misattribution is common in sports. The football athlete may attribute concussion-related headaches to "stress", dehydration, or a tight fitting helmet. In athletes who have had ongoing and usually mild post-concussive symptoms, these symptoms may be viewed by the athlete as his or her baseline level of functioning. This situation is often seen when an athlete has had multiple concussions without recovery, and symptom emergence (with even light contact or exertion) has become so commonplace that the symptom is viewed as a normal and expected part of sport participation. In addition, some athletes may not be aware that symptoms such as fatigue and sleep disturbance are of a post-concussive nature, and these symptoms are often attributed to stress, too little sleep, or to a variety of other factors. Finally, some athletes may simply be asymptomatic but may still be experiencing neurocognitive deficits associated with concussion. Concussion may present in a variety of ways, depending on the nature of the injury, the athletes history of injury, family medical background (e.g. history of migraine) or the pre-injury history of disorders such a headache or learning disability. Thus not all athletes experience symptoms such as headache, dizziness or nausea. The imperfect relationship between self-reported symptoms and neurocognitive test performance underscores the importance of neuropsychological testing and the importance of careful evaluation of symptoms and neurocognitive assessment for concussion management.

We believe that the findings of this study have direct relevance to clinical care of the concussed athlete. First, this study indicates that the evaluation of self reported symptoms may be inadequate in diagnosing concussion and in evaluating recovery. Clinical practice should include neuropsychological testing whenever possible. In addition, neuropsychological testing can be used to identify cognitive difficulties which can be used to 
devised academic accommodations that will assist the transition of the injured athlete back into the classroom during recovery.

There are limitations to the study. First, the sample for this study consisted of a sample limited to high school and collegiate athletes, who were primarily males. These characteristics of the sample limit the generalizability of the findings to professional athletes and to younger children. Given recent research linking age to differences in neurological recovery [14] and known neurometabolic differences between older and younger athletes [2], further research is needed to evaluate this group. Furthermore, the interaction of gender, symptoms, and neurocognitive performance was not examined and therefore conclusion about gender differences can not be made based on this largely male sample. Therefore, future research directions would include examining neuropsychological testing and the recovery process in different populations including younger children, women athletes, and professional athletes.

The results of the present study indicate that symptom report alone is not sufficient information for a clinician to manage concussion. From the data presented in this study as well as previously published data, it is reasonable to assume that a portion of athletes who are asymptomatic after concussion may still exhibit neurocognitive deficits on testing, and therefore should be held from play until those difficulties resolve. Our current findings again provide support for using objective measures of recovery, in conjunction with a thorough clinical and symptom evaluation as outlined in the Vienna and Prague conferences.

\section{References}

[1] AAN, Practice parameter: the management of concussion in sports (summary statement), Report of the Quality Standards Subcommittee, Neurology 48(3) (1997), 581-585.

[2] E.F. Aldrich, H.M. Eisenber, C. Saydjari, T.G. Luerssen, M.A. Foulkes and J.A. Jane, Diffuse brain swelling in severely head injured children, Journal of Neurosurgery (6) (1992), 450454.

[3] M. Aubry , R.C. Cantu, J. Dvorak et al., Summary and agreement statement of the 1st International Symposium on Concussion in Sports, Vienna, Clinical Journal of Sport Medicine 12(1) (2002), 6-11.

[4] J.E. Bailes and R.C. Cantu, Head injury in athletes, Neurosurgery 48(1) (Jan 2001), 26-45; discussion 45-26.

[5] J.E. Bailes, M.R. Lovell and J.C. Maroon eds, Sports Related Concussion, Quality Medical Publishing, St. Louis, 1999.

[6] J.T. Barth, W.M. Alves, T.V. Ryan, S.N. Macciocchi, R.W. Rimel, J.A. Jane and W.E. Nelson, Mild head injury in sports: Neuropsychological sequelae and recovery of function, in:
Mild Head Injury, H.S. Levin, H.M. Eisenberg, M. Howard and A.L. Benton, eds, Oxford University Press, New York, 1989, pp. 257-275.

[7] R. Cantu and R. Voy, Second Impact Syndrome: A risk in any sport, Physical Sports Medicine 23 (1995), 27-36.

[8] A.Collie, P. Maruff, M. Makdissi, P. McCrory, M. McStephen and D. Darby, CogSport: Reliability and correlation with conventional cognitive tests used in post concussion medical evaluation, Clinical Journal of Sport Medicine (13) (2003), 28-32.

[9] M.W. Collins, M. Field, M.R. Lovell, G.L. Iverson, K.M. Johnston, J. Maroon et al., Relationship between postconcussion headache and neuropsychological test performance in high school athletes, American Journal of Sports Medicine 31(2) (2003), 168-173.

[10] M.W. Collins, S.H. Grindel, M.R. Lovell et al., Relationship between concussion and neuropsychological performance in college football players, JAMA (10) (Sept 8, 1999), 964-970.

[11] R.J. Echemendia, M. Lovell and J. Barth, Neuropsychological Assessment of Sports-Related Mild Traumatic Brain Injury, in: Clinical Neuropsychology and Cost Outcome Research: A Beginning, G.P. Prigatano and N.H. Pliskin eds, Psychological Press, East Sussex, 2003, pp. 351-369.

[12] R.J. Echemendia, M. Putukain, R.S. Makin, L. Julian and N. Shoss, Neuropsychological test performance prior to and following sports-related mild traumatic brain injury, Clinical Journal of Sports Medicine 11(1) (Jan 2001), 23-31.

[13] D. Erlanger, T. Kaushik, R.C. Cantu, J. Barth,, D.K. Broshek, J.R. Freeman and F.M. Webbe, Symptom based assessment of severity of concussion, Journal of Neurosurgery (98) (2003), 477-484.

[14] M. Field, M.W. Collins, M.R. Lovell et al., Does age play a role in recovery from sports related concussion? A comparison of high school and collegiate athletes, Journal of Pediatrics 142(5) (May 2003), 546-553.

[15] K.M. Guskiewicz, M. McCrea, S.W. Marshall, R.C. Cantu, C. Randolph, W. Barr, J.A. Onate and J.P. Kelly, Cumulative effects associated with recurrent concussion in collegiate football players: The NCAA concussion study, JAMA 290(19) (2003), 2549-2555.

[16] D.A. Hovda, M. Prins and D.P. Becker, Neurobiology of concussion, in: Sports-Related Concussion, J.E. Bailes, M.R. Lovell and J.C. Maroon, eds, Quality Medical Publishing, St. Louis, 1999, pp. 12-15.

[17] G.L. Iverson and M. Gaetz, Practical Considerations for interpreting change following brain injury in Traumatic Brain Injury in Sports: An International neuropsychological perspective, in: Traumatic Brain Injury in Sports, M. R. Lovell, E.J. Echemendia, J.T. Barth and M.W. Collins, eds, Swets \& Zeitlinger, Lisse, 2004, pp. 323-356.

[18] G.L. Iverson, M. Gaetz, M.R. Lovell and M.W. Collins, Relation between subjective fogginess and neuropsychological testing following concussion, Journal of the International Neuropsychological Society 10 (2004), 1-3.

[19] G.L. Iverson, M. Lovell and M.W. Collins, Validity of ImPACT for measuring processing speed following sports-related concussion, Journal of Clinical and Experimental Neuropsychology, (in press).

[20] G.L. Iverson, M.R. Lovell and M.W. Collins, Interpreting change on ImPACT following sport concussion, The Clinical Neuropsychologist 17(4) (November 2003), 460-467.

[21] M.R. Lovell, Neuropsychological assessment of the professional athletes, in: Sports-Related Concussion, J.E. Bailes, M.R. Lovell and J.C. Maroon, eds, Quality Medical Publishing, St. Louis, 1999, pp. 200-214. 
[22] M.R. Lovell and C. Burke, Neuropsychological testing in ice hockey, in: Neurologic Disorders of the Head and Spine, R. Cantu, ed., Saunders, Philadelphia, 2000, pp. 109-116.

[23] M. Lovell and M.W. Collins, Neuropsychological assessment of the college football player, Journal of Head Trauma Rehabilitation 13(2) (Apr 1998), 9-26.

[24] M.R. Lovell, M.W. Collins, G.L. Iverson et al., Grade 1 or ding concussions in high school athletes, American Journal of Sports Medicine 32(1) (Jan-Feb 2004), 47-54.

[25] M.R. Lovell, M.W. Collins, G.L. Iverson, M. Field, J.C. Maroon and R. Cantu et al., Recovery from mild concussion in high school athletes, Journal of Neurosurgery 98(2) (2003), 296-301.

[26] M. Lovell, M. Collins, J. Maroon et al., Inaccuracy of symptom reporting following concussion in athletes, MMSE 34(5) (May 2002), S298.

[27] M.R. Lovell, M.W. Collins, K. Podell, J. Powell and J. Maroon, ImPACT: Immediate Post-Concussion Assessment and Cognitive Testing, Pittsburgh, PA: NeuroHealth Systems, LLC (2000).

[28] M.R. Lovell, G.L. Iverson, M.W. Collins, K. Podell, D. Pardini, J. Stump, J. Norwig and J.C. Maroon, Measurement of symptoms following sports-related concussion: Reliability and normative data for the Post-Concussion Scale, Applied Neuropsychology, (in press).

[29] J.C. Maroon, M.R. Lovell, J. Norwig et al., Cerebral concussion in athletes: evaluation and neuropsychological testing, Neurosurgery 47(3) (Sept 2000), 659-669; discussion 669672.

[30] M. McCrea et al., Acute effects and recovery time following concussion in collegiate football players: The NCAA concussion study, JAMA (290) (2003), 2556-2563

[31] P.R. McCrory and S.F. Berkovic, Video analysis of acute motor and convulsive manifestations in sport-related concussion, Neurology (54) (2000), 1488-1491.

[32] P. McCrory, K. Johnston, W. Meeuwisse et al., Summary and agreement statement of the 2nd International Conference on Concussion in Sport, Prague 2004, Clinical Journal of Sports Medicine 15(2) (March 2005), 48-55.

[33] J.P. Mihalik, J.E. Stump, M.W. Collins, M.R. Lovell, M. Field and J.C. Maroon, Posttraumatic migraine characteristics in athletes following sports-related concussion, Journal of Neurosurgery (102) (2005), 850-855.
[34] R.S. Moser, P. Schatz and B. Jordan, Prolonged effects of concussion in high school athletes, Clinical Neurosurgery (57) (2005), 300-306.

[35] E.J. Pellman, M.R. Lovell, D.C. Viano and I.R. Casson, MTBI in professional football: Recovery in NFL and high school athletes - Part 12, (in press).

[36] E.J. Pellman, M.R. Lovell, D.C. Viano, I.R. Casson and A.M. Tucker, Concussion in professional football: neuropsychological testing - part 6, Neurosurgery 55(6) (December 2004), 1290-1305.

[37] E.J. Pellman, D.C. Viano, I.R. Casson, C.A. Arfken and J. Powell, Concussion in professional football: injuries involving 7 or more days out - Part 5, Neurosurgery 55(5) (Nov 2004), 1100-1119.

[38] E.J. Pellman, D.C. Viano, I.R. Casson, A.M. Tucker, J.F. Waeckerle, J.W. Powell and H. Feuer, Concussion in professional football: repeat injuries - part 4, Neurosurgery 55(4) (Oct 2004), 860-873; discussion 873-866.

[39] C.L. Peterson, M.S. Ferrara, M. Mrazik, S. Piland and R. Elliott, Evaluation of neuropsychological domain scores and postural stability following cerebral concussion in sports, Clinical Journal of Sports Medicine (13) (2003), 230-237.

[40] K. Podell, Computerized assessment of sports-related brain injury, in: Traumatic Brain Injury in Sports, M.R. Lovell, E.J. Echemendia, J.T. Barth and M.W. Collins, eds, Swets \& Zeitlinger, Lisse, 2004, pp. 375-393.

[41] L.D. Ravdin, W. Barr, B. Jordan, W. Lathan and N. Relkin, Assessment of cognitive recovery following sports related had trauma in boxers, Clinical Journal of Sports Medicine (13) (2003), 21-27.

[42] P. Schatz, J.E. Pardini, M.R. Lovell, M.W. Collins and K. Podell, Sensitivity and specificity of the ImPACT test battery for concussion in athletes, Archives of Clinical Neuropsychology 21 (2006), 91-99.

[43] Statistical Package for the Social Sciences (SPSS). Chicago. 2005.

[44] D.A. VanKampen, M.R. Lovell, J.E. Pardini, M.W. Collins and $\mathrm{F}$. Fu, The value added of neurocognitive testing following sports-related concussion, American Journal of Sports Medicine (in press). 